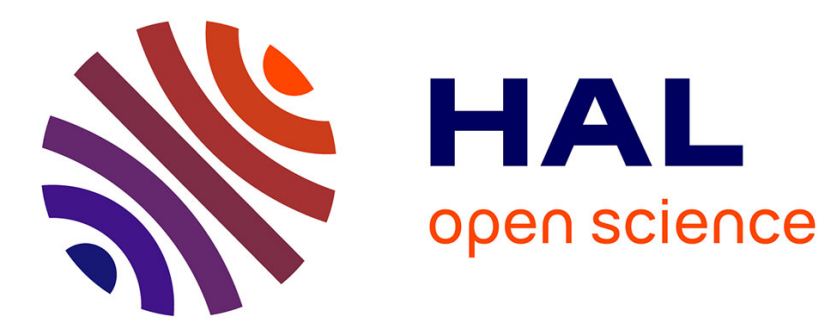

\title{
Équipes, bandes, classes d'âges : la vie juvénile de cité et de rue sous forme de poupées russes
}

Thomas Sauvadet

\section{To cite this version:}

Thomas Sauvadet. Équipes, bandes, classes d'âges: la vie juvénile de cité et de rue sous forme de poupées russes . Marwan Mohammed, Laurent Mucchielli. Les bandes de jeunes. Des " blousons noirs

" à nos jours, La Découverte, pp.123-144, 2007, 9782707153456. hal-01330871

\section{HAL Id: hal-01330871 \\ https://hal.science/hal-01330871}

Submitted on 13 Jun 2016

HAL is a multi-disciplinary open access archive for the deposit and dissemination of scientific research documents, whether they are published or not. The documents may come from teaching and research institutions in France or abroad, or from public or private research centers.
L'archive ouverte pluridisciplinaire HAL, est destinée au dépôt et à la diffusion de documents scientifiques de niveau recherche, publiés ou non, émanant des établissements d'enseignement et de recherche français ou étrangers, des laboratoires publics ou privés. 
Thomas Sauvadet, «Équipes, bandes, classes d'âges : la vie juvénile de cité et de rue sous forme de poupées russes », dans Mohammed M., Mucchielli L. (dir.), Les bandes de jeunes. Des « blousons noirs » à nos jours, Paris, La Découverte, 2007, pp. 123-144.

\section{Equipes, bandes, classes d'âges :}

\section{la vie juvénile de cité et de rue sous forme de poupées russes}

Qui sont les «jeunes de cité »? Est-ce qu'il suffit d'habiter une cité HLM pour être un «jeune de cité »? Quelles sont les spécificités des jeunes qui, en plus d'habiter en HLM, traînent dans les rues de leur quartier ? Quels types de groupe arrivent-ils à créer ?

Cet article décrit les différents modes de regroupement et d'association entre pairs des jeunes dits « de cité », ainsi que la façon dont ils se hiérarchisent, collaborent ou s'opposent.

Il analyse également les rapports de concurrence et de solidarité entre les plus jeunes et les plus âgés dans le cadre de « la vie juvénile de rue et de cité ».

1) Présentation méthodologique et présentation des terrains d'enquête

Trois monographies ont été réalisées pour cette recherche basée sur l'observation participante ${ }^{1}$. La première a été effectuée dans une cité HLM de la banlieue sud de Paris où j'ai passé une partie de ma jeunesse et où j'ai rencontré la plupart des jeunes qui «trânent » dans les rues. Ma présence y était devenue naturelle. La seconde monographie a été menée dans une cité de la banlieue nord parisienne et la dernière à Marseille ${ }^{2}$. Sur ces deux autres terrains, j'ai essayé de reproduire l'approche qui avait rendu possible mon intégration, sur Paris sud, à la vie juvénile de rue, supposant une certaine similitude.

Il fallait d'abord, pour cela, habiter sur place. A cette époque, je venais d'emménager dans un petit pavillon ouvrier situé juste en face de Paris nord, d'où son choix comme terrain d'enquête. Le terrain marseillais s'est imposé en raison des réseaux familiaux et amicaux qui pouvaient m'aider dans ma démarche. J'ai ensuite cherché à m'introduire dans les groupes de jeunes de rue, aisément repérables : comme sur Paris sud, des jeunes erraient sur l'espace public, se regroupaient et se présentaient comme les «jeunes de la cité » («du quartier » à

\footnotetext{
${ }^{1}$ L'anonymat (des lieux et des noms) a été adopté pour éviter la stigmatisation des quartiers concernés par l'enquête et pour garantir une protection aux jeunes engagés dans une carrière délinquante.

2 Dans la suite du texte, ces terrains s'appelleront « Paris sud », « Paris nord » et « Marseille ».
} 
Marseille). Mon introduction se faisait systématiquement par l'intermédiaire de l'un d'entre eux. Je disposais toujours d'un « informateur-médiateur » : comme informateur, il raconte son parcours et donne sa vision des choses, comme médiateur, il introduit, présente et protège. Qui ont été mes «informateurs-médiateurs »?

A Paris nord, une amie connaissait bien l'un des jeunes, Samir, qui se trouve être l'un des principaux délinquants des environs. Sur Marseille, Pascal, un ami de dix ans, m'a beaucoup aidé. Lors de l'enquête, il animait une association locale de boxe. Le problème principal était la méfiance que nourrissait le leadership délinquant à mon égard. Les techniques de déstabilisation, d'enquête sur l'enquêteur, ont fait partie du détour obligé, d'autant plus que les trafics qui animent les rues de ce quartier se montrent nettement plus structurés et rentables que ceux des terrains parisiens.

Pour être accepté, il convient de ne pas montrer des signes trop visibles de peur et/ou de naïveté, tout en restant modeste. Il s'agit de trouver la «bonne distance » tout en rendant des services constants (véhiculer, prêter une cassette vidéo...) et en effectuant des dons divers (cigarettes, cafés...) afin de constituer une sorte de capital sympathie, convertible en droit d'observer. Vocabulaire, attitudes gestuelles, style vestimentaire, sont autant de dimensions à maîtriser pour jouer la carte de la complicité et ne pas perturber les interactions ordinaires, « se fondre dans le décor» (ce qui n'est vraiment possible que pour un sociologue jeune, de sexe masculin et un minium aguerri). Cette complicité impose toutefois certaines limites à l'enquête, comme la difficulté d'accéder aux relations familiales: pour des raisons compréhensibles, les enquêtés séparent l'univers de la rue de celui de la famille.

Mon observation participante s'est étalée de manière discontinue entre 2000 et 2003, avec presque deux ans de présence régulière sur Paris nord, six mois de présence quotidienne sur Marseille et un suivi de Paris sud, terrain pour lequel mes prises de notes avaient commencé dès 1998.

Sur ces trois terrains, nous constatons un fort taux de chômage (le double de la moyenne nationale), beaucoup d'ouvriers et de petits employés, de travailleurs précaires et de ménages surendettés. Paris nord, 1500 habitants, est deux fois plus petit que Paris sud, lui-même presque deux fois moins grand que Marseille. A Paris nord, les cités ne sont séparées les unes des autres que par une poignée de pavillons et nous constatons régulièrement des conflits entre bandes de quartiers rivaux. La cohabitation apparaît difficile, la ville, surpeuplée. A Paris sud, la forêt et quelques zones pavillonnaires encerclent la cité. Le cadre de vie semble plus agréable bien que plus enclavé. Les conflits entre jeunes de cités rivales sont fréquents. A Marseille, nous retrouvons une forte densité de population, mais avec un tissu associatif de 
qualité, une habitude du brassage ethnique et culturel, elle-même conjuguée à une identité locale populaire affirmée qui limite la stigmatisation des attitudes populaires et donc les frustrations et la mésestime de soi des jeunes de rue. Les conflits entre bandes de quartiers différents n'entraînent pas ici des batailles rangées. Le terrain marseillais, contrairement aux deux autres, n'a pas « brûlé » en novembre $2005^{3}$.

2) Qui sont les «jeunes de cité »? Ceux de la rue, l'espace du pauvre...

La structure démographique de ces cités correspond presque à celle des pays pauvres : la population âgée de moins de 30 ans représente aux alentours de $50 \%$ de la population totale. La rue est jeune, la présence adulte, faible. Au début des années 1980, dans un bassin minier vieillissant du Nord - Pas-de-Calais, Olivier Schwartz constate que «l'enrichissement » de la classe ouvrière dans les années 1950, 1960 et 1970 a produit un investissement plus important dans la vie domestique, dans l'espace privé, un « espace féminin $»^{4}$. De nombreuses familles, comme c'est aussi le cas sur mes terrains d'enquête, ont ainsi limité leur insertion, jadis «naturelle », dans la communauté du quartier, et ont de ce fait rompu avec les modes de vie des anciens. Comme le remarque Olivier Schwartz, le chômage structurel des années 1980 a ensuite transformé le foyer en un lieu de repli nécessaire pour «sauver la face ». La montée de l'insécurité réelle et fantasmée a également induit une mise à distance de la vie sociale locale. Par ailleurs, beaucoup de parents ont connu les épreuves de l'émigration/immigration observées par Abdelmalek Sayad ${ }^{5}$ et doutent de la légitimité de leur parole dans la sphère publique.

Si la rue est jeune, seuls les «jeunes de la cité » sont présents dans les rues : soit environ $10 \%$ de la population masculine de moins de trente ans ${ }^{6}$. Pour les autres, et notamment pour la

\footnotetext{
3 Pour une approche comparative détaillée, voir: Thomas Sauvadet., «Les jeunes « de la cité » : comment forment-ils un groupe? Une analyse comparative entre trois terrains », Socio-logos, ${ }^{\circ} 1$, juin 2006, http://sociologos.revues.org/document55.html.

4 Malgré ce repli sur la sphère domestique, Olivier Schwartz est attentif aux efforts masculins accomplis pour desserrer l'étau, rompre l'enfermement sur l'espace domestique, esquisser un ailleurs, ne serait-ce que sous la forme d'un refuge onirique. À ce sujet, voir par exemple la deuxième partie du chapitre IV : «Les espaces de recomposition masculine » (Olivier Schwartz, Le monde privé des ouvriers. Hommes et femmes du Nord, Paris, PUF, 2002).

5 Abdelmalek Sayad, La double absence : des illusions de l'émigré aux désillusions de l'immigré, Paris, SeuilLiber, 1999.

6 Au début des années 1980, Jean-Charles Lagrée et Paula Lew-Fai observaient déjà le même phénomène et constataient que la représentation collective de la jeunesse locale passait sous silence 93\% des jeunes ( $L a$ galère : marginalisations juvéniles et collectivités locales, Paris, CNRS, 1985).
} 
quasi totalité des filles (à l'exception des enfants)7 ${ }^{7}$, la cité ne représente qu'un lieu de passage, une «cité dortoir » et, en général, ces jeunes souhaitent se distinguer de ceux de la rue, ces garçons qui «traînent» et qui symbolisent la déviance voire la délinquance. Cette grande majorité «d'invisibles » forme un vaste champ d'enquête que ce travail ne fait qu'effleurer. Les principales raisons de cette absence semblent reposer sur les dimensions suivantes : 1) l'absence d'activités jugées suffisamment stimulantes ; 2) la cohabitation difficile des groupes de jeunes constamment présents dans l'espace public ; 3) la stigmatisation subie par les jeunes qui fréquentent cet espace («voyous», «sauvageons», "fainéants») discrédite ce type de socialisation symbole de marginalisation sociale ${ }^{8}$; 4) la possibilité de s'inscrire dans d'autres univers sociaux (activité professionnelle, «cocooning», vie conjugale, vacances...), largement dépendante du capital familial économique et culturel.

Souvent originaires des anciennes colonies françaises, les garçons « des rues » proviennent massivement des familles les plus pauvres: ils partent rarement en vacances, n'ont pas d'argent de poche et beaucoup d'entre eux partagent leur chambre avec leur(s) frère(s). Les 20/35 ans ne disposent que d'un CAP, de « tourneur-fraiseur », au mieux d'un BEP, « vente », et les trois-quarts cherchent un emploi. L'intérim, les stages de formation sous-payés, le travail au noir et les CDD offrent de maigres « opportunités professionnelles ${ }^{9}$ ». Quelques-uns goûtent aux espoirs de la massification scolaire et atteignent le niveau universitaire avant de connaître les désillusions analysées par Stéphane Beaud ${ }^{10}$.

$\mathrm{Si}$ «traîner dans les rues » dès le plus jeune âge renvoie systématiquement à une condition sociale défavorisée, provenir d'une famille pauvre (comparativement aux autres familles du quartier et à la société française en général) n'est pas automatiquement lié au fait de «traîner » dans les rues de son quartier. Ainsi, à Paris sud, un jeune vietnamien préfère vendre de la cocaïne dans le $13^{\text {ème }}$ arrondissement de Paris (où est rassemblée une importante

\footnotetext{
${ }^{7}$ La vie juvénile de rue est essentiellement masculine, mais elle ne l'est pas exclusivement, je dirais même qu'elle l'est de moins en moins. Les groupes de jeunes ici étudiés sont exclusivement masculins mais peuvent cohabiter avec quelques groupes féminins. Pour plus de précisons voir par exemple : Stéphanie Rubi, Les "Crapuleuses », ces adolescentes déviantes, Paris, PUF, 2005.

8 Lors de son enquête dans la cité du Luth de Gennevilliers, Olivier Masclet écrit : «Beaucoup de parents «redoutent par-dessus tout que leurs enfants (...) s'habillent, s'amusent et parlent comme ceux qui tiennent la rue » (La gauche et les cités. Enquête sur un rendez-vous manqué, Paris, La Dispute/SNEDIT, 2003). Ainsi, le travail célèbre de Jean-Claude Chamboredon et de Marianne Lemaire, qui montre l'hétérogénéité sociale des cités HLM et les logiques de distinction qui en découlent, reste d'actualité ; $c f$. «Proximité spatiale et distance sociale. Les grands ensembles et leur peuplement », Revue française de sociologie, Vol. XI, n, janvier-mars 1970.

${ }^{9}$ Voir à ce sujet le travail précurseur de Michel Pialoux au titre évocateur : «Jeunesse sans avenir et travail intérimaire », Actes de la recherche en sciences sociales, $\mathrm{n}^{\circ} 26-27,1979$.

10 Stéphane Beaud, $80 \%$ au bac... et après? Les enfants de la démocratisation scolaire, Paris, La Découverte, 2002 .
} 
communauté asiatique) et se montre remarquablement mobile. Un autre jeune prend le contrepied de son grand frère qui a «mal tourné », réussit ses examens scolaires et fait le bonheur de ses parents ${ }^{11}$. Un autre adhère aux Témoins de Jéhovah, un autre à un groupe de militants islamiques. Une carrière sportive pleine de rêves de gloire absorbe tel ou tel jeune...

« Traîner dans les rues » n'est pas un acte anodin, il tend à indiquer une position sociale, celle de la marginalisation, la voie du danger voire du « péché » pour certains religieux.

La population juvénile et masculine qui «traîne » s'avère elle aussi hétérogène. «Avec cette ambivalence qui imprègne l'attachement de l'individu pour sa catégorie stigmatique, on conçoit que ce n'est pas toujours sans vacillation qu'il la soutient, s'y identifie et y participe. Il y a ainsi tout un "cycle de l'affiliation", suivant lequel l'individu en vient à accepter les occasions qui s'offrent à lui de participer au groupe, ou bien à les rejeter alors qu'il les acceptait auparavant ${ }^{12}$. $\gg$ Les conflits familiaux ${ }^{13}$, l'échec scolaire et professionnel, les discriminations diverses (entrées en discothèque...), l'échec amoureux, les gratifications tirées de la délinquance, la solidarité des copains de la rue, etc., créent des forces centripètes, dans le sens où ils renforcent ou entérinent l'affiliation à la catégorie stigmatique en renvoyant - directement ou indirectement - à cette catégorie. Parallèlement s'exercent des forces centrifuges (obtention d'un emploi en contrat à durée indéterminée, escroqueries et bagarres entre copains «de la cité», relation amoureuse, renouveau de l'influence parentale $\left.{ }^{14} \ldots\right)$.

Ces forces centripètes et centrifuges engendrent une diversité de positions. Certains jeunes se disent des «purs produits de la cité » car ils y passent le plus clair de leur temps et ne voient pas d'alternative à cette situation. D'autres se trouvent proches de la «majorité invisible » évoquée précédemment. Ils viennent souvent de familles moins fragilisées sur le plan socio-

11 Sur les mécanismes d'élection parentale et leurs implications sur l'élection scolaire, voir Gérard Mauger, «Election parentale, élection scolaire», dans Parents et adolescents, des interactions au fil du temps, Paris, Erès, 2001.

12 Erving Goffman, Stigmate, Paris, Minuit, 1975.

$13 \mathrm{Si}$ mettre à la porte du domicile familial les jeunes rétifs au travail a longtemps représenté la seule solution offerte aux pères ouvriers pour contraindre leur fils à se prendre en charge, cette stratégie est aujourd'hui compromise par la pénurie réelle de travail, et donc par la menace d'une condamnation à l'errance (Numa Murard, «Autorité et amour : éducation des enfants ou mise en condition?», Mouvements, $\mathrm{n}^{\circ} 8$, mars-avril 2000). En conséquence, la froideur des relations familiales renvoie souvent aux évitements multiples qui conditionnent le maintien de rapports pacifiés.

14 Laurent Mucchielli («Familles et délinquances. Un bilan pluridisciplinaire des recherches francophones et anglophones », CESDIP, Etudes et données pénales, n 86,2000 ) observe que toutes les études sérieuses tendent à montrer que les ressources socio-économiques conditionnent le niveau de contrôle parental, or celui-ci joue un rôle majeur dans le rapport que l'enfant, puis l'adolescent et le jeune homme, entretiennent avec la rue. La surpopulation du domicile familial, signe du manque cruel d'argent, incite par exemple les enfants à quitter le foyer familial (et donc à s'éloigner du contrôle parental), mais leur faible pouvoir d'achat rend les «sorties » compliquées. Il reste la rue. 
économique (ils disposent par exemple d'une chambre personnelle et leur scolarité dépasse le cap des 16 ans) et sont souvent perçus comme des "fils à papa» qui cherchent à «s'encanailler » de façon temporaire.

Les «purs produits de la cité » se différencient les uns des autres. Certains dominent, d'autres jouent les seconds rôles. Certaines périodes de vie se caractérisent par des forces centripètes, d'autres par des forces centrifuges, même si, en définitive, la faiblesse de ces dernières induit une identité revendiquée de "pur produit de la cité »15.

La vie juvénile de rue se compose d'une population qui va de cinq à 30/35 ans et cette sociabilité s'apparente par moments à des rapports de filiation qui se manifestent à travers des expressions comme "petits frères », «filstons », «les grands », etc. Néanmoins, les enquêtés fréquentent essentiellement leurs pairs et s'associent avec eux pour former des groupes rassemblant l'ensemble ou une partie de ces derniers. Toutes ces démarcations ne sont pas toujours explicitées, il n'y a pas de formalisme, les rencontres s'effectuent régulièrement sur un mode aléatoire et les appartenances peuvent être floues et multiples, notamment pour les groupes d'enfants.

\section{3) Les principaux modes de regroupement}

J'ai observé, à côté du caractère aléatoire des rencontres, trois logiques principales de regroupement qui renvoient à différents niveaux d'association entre pairs. La première, certains jeunes la nomment «l'équipe». La seconde, nous l'appellerons « la bande ». La dernière correspond à une "classe d'âge ", j'en différencie au minimum trois : l'enfance (jusqu'à dix ans), l'adolescence (jusqu'à vingt ans) et le jeune âge adulte (jusqu'à 30/35 ans).

Les vrais potes : l'équipe

L'équipe se définit comme le plus petit groupe de pairs observable. Avec la fratrie, elle incarne la pierre fondatrice de la vie juvénile de rue. Les interdépendances affectives et économiques y sont cruciales. On y retrouve les vrais «potes », à l'inverse des faux qui se glissent dans les cercles relationnels moins exclusifs. Les équipes tendent à se former dès l'enfance et se caractérisent par une forte homogénéité (affinités caractérielles, proximité liée à l'âge, équivalence des ressources familiales...). 
Les enfants se socialisent sur l'espace public de leur cité et découvrent tous ceux de leur âge qui y évoluent, puis, ou conjointement, en retrouvent certains dans le cadre scolaire : cette double proximité favorise le développement de la camaraderie. Le centre social et les terrains de sport jouent aussi un rôle important ${ }^{16}$.

Sur Paris sud et Marseille, du fait de la dimension plus importante de ces quartiers, la proximité géographique des habitations familiales conditionne la production des équipes. Sur Paris sud, la sectorisation scolaire coupe la cité en deux : de la maternelle jusqu'au lycée, les enquêtés ne sont pas scolarisés dans les mêmes établissements. Cette situation a engendré des conflits entre les jeunes de chaque zone de sectorisation.

À l'adolescence, la connaissance approfondie de la cité offre du même coup un large choix de copains aptes à entrer dans la composition d'une équipe. Cette composition dépend systématiquement de celle de la bande de référence. L'association de plusieurs équipes donne naissance à une bande.

Tour d'horizon des bandes rencontrées

Sur Paris sud, une bande de jeunes adultes se nomme «Criminel Boys » et y associe le nom de la cité, l'ensemble se prononce sous forme de sigle ${ }^{17}$.

Composée d'une quinzaine de personnes, la bande des « $\mathrm{CB} »$ s'est formée autour de deux leaders charismatiques : Farouk, alias Grand Farouk, et Aziz. Cette bande se retrouve près de chez Grand Farouk, celui-ci constitue un pôle d'attraction : ses copains, et ceux qui cherchent à l'être, viennent le trouver à cet endroit. Leur bande exerce une forte influence sur les autres groupes de jeunes. Lorsqu'elle «débarque » quelque part, les lieux lui appartiennent. Le centre social et les terrains de sport sont le théâtre privilégié de ce genre d'appropriation (et d'expropriation). Les jeunes qui composent les $\mathrm{CB}$ se connaissent pour la plupart depuis l'enfance. Ils sont presque tous originaires du Maghreb, si bien que ceux qui ne le sont pas mettent en avant un ancêtre maghrébin, parfois plus ou moins imaginaire, et apprennent un peu de vocabulaire arabe. Au début des années 1990, le nom «Criminel Boys » a été adopté. À cette époque, les adolescents concernés sont reconnus comme des voleurs d'exception et

15 Pour une analyse détaillée de cette typologie, voir : Thomas Sauvadet, Le Capital guerrier : Solidarité et concurrence entre jeunes de cité, Paris, Armand Colin, 2006.

16 Olivier Masclet (2003, op. cit.) souligne l'importance que revêt la socialisation au sein d'un club de jeunes dans le dépassement des hostilités qui peuvent opposer des groupes distincts et construits sur des bases microterritoriales, mais précise que la rue demeure le premier espace des rencontres. 
attirent l'attention en exécutant des acrobaties avec des motos volées. Par la suite, Grand Farouk et Aziz deviennent les principaux grossistes de cannabis du quartier et accroissent l'influence des CB. À partir de la fin des années 1990, leur bande abrite une «équipe » de rappeurs confirmés, la Mafia du Mic (du Microphone). En 1999, la bande se scinde en deux après un conflit financier entre Farouk et Aziz. Le groupe d'Aziz se déplace vers le café du coin.

L'autre bande de cette classe d'âge se compose d'une douzaine de membres et se prénomme les «KBA », sans que les jeunes puissent expliquer la signification de ce sigle, les versions sont multiples et confuses. Cette bande s'est constituée parallèlement aux $\mathrm{CB}$, en représentant un pôle de socialisation moins délinquant, moins marqué ethniquement et plus axé sur la consommation de drogues douces. Son point de rencontre le plus habituel correspond au lieu d'habitation de certains membres du groupe, à distance du squat des CB. Les KBA et les CB ont néanmoins toujours été intimement liés. Le leader des KBA est un transfuge des $\mathrm{CB}$ : suite à une bagarre perdue, il a rejoint les KBA puis en a pris le contrôle. Son petit frère est resté $\mathrm{CB}$. Ces deux bandes effectuent de nombreuses activités en commun, elles partagent notamment un sous-sol qu'elles aménagent comme elles peuvent : banquettes, tables, chaises, etc., sont récupérées dans les poubelles.

Chez les adolescents, un groupe d'une vingtaine d'individus se nomme la «Bédo Connexion » (bédo signifie cannabis). Comme les plus âgés, ces jeunes y associent le nom de la cité et prononcent l'ensemble sous forme de sigle. Celui-ci fait d'ailleurs référence au célèbre « $\mathrm{CB}$ », dont ils ont connu la cohésion et dont ils disent assumer la relève. Cette bande existe depuis une dizaine d'années et s'est spécialisée dans la consommation et la revente de cannabis. Son point de rencontre se trouve sur une petite place nommée «les pierres ». Un sous-sol aménagé sert de repaire.

L'autre bande de cette classe d'âge se compose d'une douzaine de membres et n'a pas d'autre nom que «la bande à Embre 18 ». Son point de rencontre se situe près d'une butte de terre à côté de laquelle ont été installés quelques bancs publics. Deux sous-sols et un hall situés à proximité sont squattés et parfois aménagés pour plus de confort. On trouve dans cette bande quelques fumeurs de cannabis mais elle contient essentiellement des délinquants/sportifs et de simples sportifs. Cette spécialisation est fièrement revendiquée face aux conduites addictives de la Bédo Connexion. Les adolescents de la bande à Embre ont pour la plupart emménagé

\footnotetext{
17 Nous verrons bientôt la fragilité de la terminologie «Criminel Boys », le lecteur ne doit pas penser que tous les jeunes de la bande en question se reconnaissent en permanence dans cette appellation.

18 Sur tous les terrains d'enquête, ils disent « la bande à » et non « la bande de ».
} 
dans la cité depuis moins de cinq ans. On peut penser que leur association s'est en partie réalisée sur la base d'une opposition aux établis de la Bédo Connexion. Le faible turn-over résidentiel produit ces établis. Dans le groupe de Paris sud, seulement un jeune sur cinq habite dans la cité depuis moins de dix ans ${ }^{19}$, les autres se proclament «natifs des lieux ».

Les groupes d'enfants se singularisent par la porosité de leurs frontières, par l'absence relative d'identité collective et de savoir-faire particuliers. Leur histoire se trouve devant elles.

Trois « bandes » n'ont pas d'autres noms que « la bande à ». La «bande à l'Apache », du nom d'un enfant de huit ans qui s'appelle en réalité Abderahmanne, se compose d'une quinzaine de gosses rarement tous réunis, sectorisés dans la même école primaire. Les dix garçons de la «bande à Boris », comme ceux de la «bande à Bobo », sont sectorisés dans l'autre école primaire du quartier.

La bande à l'Apache domine ses consœurs. La bande à Bobo rivalise avec la bande à Boris, avec laquelle elle s'allie parfois pour résister aux intrusions intempestives de la bande à l'Apache. La bande à Bobo se caractérise par une forte dimension ethnique (Afrique subsaharienne). Elle s'est constituée autour de Bobo et de sa fratrie : trois petits frères qui le suivent «comme son ombre » et, dès qu'ils pressentent un danger, s'alignent derrière lui par ordre décroissant de taille, ce qui fait rire les plus âgés qui les surnomment «les Daltons ». Ces enfants plus pauvres que les autres sont constamment exposés aux turbulences de la rue, à ses privations, ses violences, et sont les seuls à ne pas porter de chaussettes en plein hiver.

Les fréquentes bagarres entre groupes d'enfants n'entraînent pas de blessures graves et recouvrent souvent, du moins au départ, une dimension ludique partagée par tous les protagonistes.

Chez les jeunes adultes de Marseille, on recense la «Hittistes Association », une bande d'une quinzaine de personnes. Hittistes est un terme arabe qui signifie «ceux qui tiennent les murs ». Association se prononce en anglais. Le mélange de l'anglais et de l'arabe séduit. Les rencontres s'effectuent autour du café ou près d'une table en bois plantée au milieu d'un espace vert. À l'écart des regards, l'endroit convient à la consommation de stupéfiants (cannabis voire cocaïne). Les fumeurs de la Hittistes Association s'enracinent dans ce microterritoire. Le nom «Hittistes Association » leur a été attribué par les autres jeunes adultes du quartier. Ils essaient depuis de retourner le stigmate en emblème et n'y arrivent qu'à moitié. Cette bande s'avère particulièrement hétérogène, s'y retrouvent des jeunes qui se connaissent depuis l'enfance et des petits nouveaux, des jeunes qui sortent peu de chez eux et des jeunes

\footnotetext{
19 Dans le groupe de Paris nord, environ un tiers, un dixième dans le groupe marseillais
} 
qui traînent constamment dehors : la consommation de drogues fait le lien. La Hittistes Association rappelle la bande des KBA en plus enclavée. Elle s'est montrée relativement facile d'accès du fait de son immobilité, de sa faible paranoïa délinquante et de son agressivité modérée.

La seconde bande représente au contraire un univers plus fermé, plus mobile et plus violent. La présence et l'aide de Pascal, mon informateur-médiateur, se sont montrées indispensables à maintes reprises pour y pénétrer. Le «Shitsquad» (le «Squad du shit ») s'incarne au travers d'une petite trentaine de jeunes craints et respectés de gré ou de force, dont la plupart se connaît depuis l'enfance. Ils ont d'abord représenté le pôle de l'action, de l'aventure et du danger pour les jeunes de leur «génération », puis le pôle de la délinquance organisée, à l'instar du rôle joué par les CB sur Paris sud. Les rencontres se déroulent au café de la cité, dans une cave aménagée ou dans un hall qui offre une vue panoramique sur l'artère centrale de la cité, un lieu stratégique qu'ils fréquentent et contrôlent en permanence. La consommation de drogues, bien que pratiquée, ne correspond pas un centre d'intérêt prioritaire. Le groupe est axé sur la délinquance d'acquisition, le sport et les acrobaties motorisées. Les businesseurs du Shitsquad se déplacent avec dynamisme et leur vigilance couvre facilement l'ensemble du quartier. Comme il a été dit, pour accéder à ce groupe, le parrainage de Pascal s'est révélé primordial. Pour justifier ma présence, Pascal était contraint à l'hyperactivité. En plus de gérer ses relations habituelles avec les gars du Shitsquad, il contrôlait leur comportement à mon égard et interrompait fréquemment ses discussions pour s'immiscer dans les miennes et me sortir d'un mauvais pas. Nos contacts avec le Shitsquad représentaient des moments de tension qui ne pouvaient durer plus d'une heure ou deux, mais une fois le contact établi, je bénéficiais d'un certain passe-droit : la bande d'adolescents la plus délinquante, « la Corpo », ne me voyait plus du même œil.

Cette bande compte une quinzaine de membres. Son nom reprend celui du quartier et y associe Corporation, un terme prononcé en anglais. En abrégé, cela donne « la Corpo ». Ce collectif domine les autres bandes de la même classe d'âge et symbolise le pôle délinquant de cette «génération » avec un mélange de délinquance d'expression et d'acquisition, les yeux rivés sur le Shitsquad. Le point de rencontre est un hall d'immeuble situé non loin de celui du Shitsquad. La cohabitation semble parfois difficile. L'agitation de la Corpo entraîne la réprobation des plus âgés qui préfèrent la discrétion pour leurs affaires. La Corpo affiche ses couleurs : Blacks, Blancs, Beurs. Les petits nouveaux qui errent dans les rues à la recherche d'un peu d'action, d'amitié et d'argent, s'y intègrent facilement à condition de surmonter les défis qui façonnent en permanence la vie du groupe : le prix à payer pour être accepté et 
reconnu n'est pas négligeable (chute lors d'une acrobatie, mauvais coup dans une bagarre...). Les moqueries s'abattent sur les plus faibles avec une férocité certaine. En réaction, la «bande du haut » s'est constituée.

Cette bande, d'une dizaine d'ados, doit son nom au fait qu'elle se positionne géographiquement plus en hauteur. Elle se compose de six lycéens qui connaissent des difficultés scolaires, développent des conduites addictives (alcool et cannabis) et se connaissent depuis l'enfance. À partir du collège, ils se mêlent aux jeunes du quartier, grâce à Bruno, un gars de la Corpo. Bruno constitue un pôle autour duquel ils s'agglutinent. Parallèlement, celui-ci prend ses distances avec la Corpo, suite à un différend avec Théodore, un des leaders. D'autres transfuges arrivent par la suite : quatre jeunes proches de Bruno qui, comme lui, ont des difficultés sociales au sein de la Corpo et dans la vie en général. Les activités de la bande du haut sont centrées sur la consommation de drogues douces. Cette bande n'a pas opposé de résistance particulière à ma recherche, à l'instar des «Dog Brothers ».

La bande des « Dog Brothers », ou « DB » en sigle, s'appelle également « Division Blindée » et compte une dizaine de jeunes. Elle s'est formée par et pour le football, d'où le terme de « division ». Cinq amis d'enfance forment le noyau du groupe, ils sont membres du même club de foot et pratiquent en parallèle leur passion sur le quartier. Le petit terrain de foot sert de point de rencontre, à l'instar du hall d'où les Dog Brothers encouragent les exploits footballistiques des plus jeunes. La DB s'est récemment enrichie de quatre nouveaux membres, quatre lycéens qui apprécient son «ambiance sportive ». Cette bande représente un pôle de socialisation où les pratiques addictives et délinquantes sont limitées à l'expérimentation. Elle s'oppose implicitement aux deux autres bandes d'adolescents et s'en tient un peu à l'écart, privilégiant ses relations avec les petits frères. Il s'agirait d'un travail de prévention. Parallèlement, ces jeunes profitent des meilleurs aspects de la vie juvénile de rue et de cité : ils sont salués par tous, achètent des marchandises «tombées du camion », participent aux fêtes improvisées...

Du côté des enfants, une «bande » d'une dizaine de minots reprend le nom de la Corpo en y rajoutant «Junior ». Les liens entre les deux groupes sont d'ordre familial (frères et cousins). Ces minots fréquentent le playground du quartier où la DB les encadre, mais traînent également sur l'artère centrale dans les pattes de la Corpo et du Shitsquad. Ils forment le groupe de gamins le plus virulent. 
La «Dream Team » fréquente exclusivement le playground. Cette petite «bande » d'une dizaine d'enfants a gardé le nom qui lui a été attribué par les ados de la DB lors d'un tournoi de foot.

Les deux derniers groupes sont qualifiées par la «bande à ». La «bande à Mickey » chahute aux alentours du centre social où elle tente de monopoliser les différents jeux mis à disposition. Elle se compose d'une douzaine de gamins rarement au complet. Elle résulte de la fusion de deux petits groupes et se tient à distance des autres «bandes». Elle se retrouve régulièrement sur un terrain de sport isolé et peu fréquenté, car en mauvais état, situé à proximité du quartier. Les gosses de la bande à Mickey ne sont pas tous des «enfants du dehors » : certains d'entre eux bénéficient d'une chambre personnelle et d'une console de jeux vidéos, ils rentrent plus tôt « à la maison » et en sortent moins fréquemment.

La dernière «bande », une demi-douzaine d'enfants, se nomme la «bande d'Abou ». Ces minots déambulent de manière opportuniste à la recherche d'une bonne rigolade, jusqu'à la nuit tombée 20 .

Les modes de production et de fonctionnement des bandes

«L'étymologie du mot «bande » renvoie à la notion de groupe pourvu d'un leader et arborant un signe distinctif qui le représente et le différencie des autres. Alors qu'au début de son histoire ce mot renvoyait à un groupe légalement organisé dans le cadre d'un système social officiel, peu à peu, la bande est devenue un groupe considéré comme marginal et dangereux $»^{21}$.

Comme l'explique Maryse Esterle-Hedibel, la dangerosité et la négativité se fixent autour du mot, d'où son utilisation sous forme d'injure : «bande de vagabonds ! ». L'effet péjoratif du terme incite les jeunes étudiés à ne pas l'employer ou uniquement pour qualifier les groupes d'adolescents et d'enfants, qu'ils diabolisent affectueusement et sur le mode de la rigolade.

Malgré les problèmes posés par le terme «bande », celui-ci représente un référent commun entre les jeunes de cité et les loubards : il désigne en un instant la similitude des modes de socialisation $^{22}$.

\footnotetext{
${ }^{20}$ Les bandes de Paris nord seront étudiées dans le paragraphe réservé aux classes d'âges car, sur ce terrain, bande et classe d'âge ne forment qu'un seul et unique espace de socialisation.

21 Maryse Esterle-Hedibel, La bande, le risque et l'accident, Paris, L'Harmattan, 1998.

22 Pour une synthèse des débats qui se déroulèrent à la fin des années 1980 et au début des années 1990 sur le thème de la disparition des bandes, voir : Maryse Esterle-Hedibel, 1998, op. cit.
} 
Pour définir cette similitude, il me faut maintenant préciser les critères qui, je pense, m'autorisent à utiliser cette terminologie.

Les bandes étudiées possèdent toutes un nom. Pendant l'enfance, celui-ci se réfère généralement à leur leadership («la bande à »), puis, à l'adolescence, à leur spécialisation (Shitsquad, Hittistes Association, etc.). Enfin, au jeune âge adulte, il correspond à un terme du passé et véhicule une dimension nostalgique voire puérile. Demander à Grand Farouk s'il appartient aux Criminel Boys le fait forcément rire. Il a depuis longtemps dépassé ce genre de mise en scène. Sa délinquance d'expression a cédé la place à une délinquance d'acquisition discrète par obligation, et puis les Criminel Boys ont disparu lors de leur scission en 1999. Même à l'apogée des CB, dans le courant des années 1990, tous les jeunes n'adoptent pas cette terminologie, car il existe d'autres façons de nommer le groupe. La plus connue, en dehors du sigle « $\mathrm{CB} »$, renvoie à l'énumération des leaders suivie de « et les autres ». La réticence de certains à utiliser l'expression «Criminel Boys » s'explique par l'angélisme dont ils doivent faire preuve devant des interlocuteurs extérieurs à la vie juvénile de rue, mais elle résulte également du manque de croyance en la cohésion du groupe, car l'existence même du groupe est constamment menacée. Les conflits et les trahisons internes provoquent des commentaires ironiques sur la soi-disant solidarité des CB. Cette qualification apparaît de plus prétentieuse et excessive, lorsque les jeunes constatent avec amertume la faible rentabilité de leurs combines, la routine de leur quotidien et la couardise de leurs copains. Dans maintes circonstances, l'expression « et les autres », plus floue, convient mieux.

Les appellations que se donnent les bandes ne vivent en réalité que par le biais des quelques jeunes qui les défendent. Le rôle de ces «entrepreneurs de nom » se montre déterminant, notamment à travers la pratique du tag. Les tagueurs «baptisent» leur groupe de pairs. Ils déposent sur les murs une signature personnelle mais réfèrent toujours celle-ci à un collectif, soit celui de la cité, soit celui du groupe de pairs, soit les deux à la fois. Ils doivent trouver un nom qui marque les esprits. Grâce à cette pratique, ils s'approprient symboliquement et durablement l'espace, celui de «leur » hall d'immeuble par exemple, s'attribuent un nom et le font savoir. Ils introduisent une façon originale de nommer le groupe et d'autres jeunes les imitent jusqu'à un certain point.

Le nom d'une bande provient parfois d'une entreprise de qualification hétéronome. Les groupes dominés, comme les groupes d'enfants ou ceux spécialisés dans la consommation de drogues, sont facilement assignés à tel ou tel qualificatif, qu'ils peuvent ensuite essayer de s'approprier, jusqu'à revendiquer le stigmate comme le fait la Hittistes Association. 
Les bandes rencontrées s'ancrent dans un territoire: selon la proximité des habitations familiales de leurs membres, selon la discrétion du site vis-à-vis des patrouilles de police et du contrôle parental, en fonction de la concurrence des autres bandes et de la permissivité des résidents, ou selon le confort et les possibilités de divertissement qu'offre l'endroit. Au-delà de cet ancrage micro-territorial, on observe un attachement au quartier. Le nom de la cité entre dans la composition du nom de la bande de manière quasi systématique. A l'époque des «blousons noirs », la territorialisation des bandes et leurs réactions agressives en cas d'affront correspondaient déjà à un sujet d'inquiétude23. Avant eux, les apaches fonctionnaient de manière identique et se prénommaient « la bande des Quatre Chemins d'Aubervilliers », «les gars de Charonne », « les Monte-en-l'air des Batignolles » ou « les Loups de la Butte ${ }^{24}$.

Les bandes se spécialisent. Le sport, la consommation de drogues (douces ou douces et dures) et la délinquance (d'expression et/ou d'acquisition) correspondent à trois activités typiques qui ne s'excluent pas forcément. Au sein d'une même classe d'âge, chaque bande constitue une sorte de pôle où se concentrent des savoir-faire spécifiques. Certains jeunes ou certaines équipes naviguent entre ces pôles et accumulent des compétences. Les bandes les plus délinquantes se composent en général des jeunes les plus pauvres, tous «purs produits de la cité », car les risques inhérents à leurs activités découragent les moins défavorisés.

Plus ouvertes que les «équipes», les bandes représentent néanmoins des groupes relativement fermés, même s'il peut être facile de les fréquenter temporairement. Pour en faire réellement partie, plusieurs épreuves informelles et plus ou moins improvisées par le reste de la bande doivent être franchies. Ces dernières dépendent évidemment de la spécialisation du groupe concerné : l'exploit délinquant détermine l'intégration aux Criminel Boys, tandis que la consommation maîtrisée de drogues constitue un point majeur de l'adaptation au mode de vie des KBA. Faouzy explique : "Hamid on le laisse en plan (seul). [...] c'est un débutant de la défonce. Il tient pas l'coup. Il part en banane (il fait n'importe quoi). C'est un boulet ce gars ».

Lorsque le «candidat » ne répond pas aux critères exigés, les mauvais traitements qu'il subit le découragent (moqueries, ordres, petites brutalités physiques, évitements, indifférences, vols...), mais certains s'accrochent.

23 Voir à ce sujet: Pierre Ceccaldi, «Le phénomène des bandes. Manifestation actuelle de la délinquance juvénile », Revue pénitentiaire et de droit pénal, n50, 1961.

24 Voir à ce sujet : Michèle Perrot, «Dans la France de la Belle Epoque, les « Apaches », premières bandes de jeunes », dans Les marginaux et les exclus dans l'histoire, Paris, UGE, 1979. 
Il existe un constat bien établi à propos de la délinquance des mineurs : le lien entre action collective et actes violents ${ }^{25}$. Selon mes observations, la bande incarne l'espace social où, par excellence, nous constatons ce lien. Pourquoi?

La bande correspond au groupe de pairs le plus fréquemment rencontré. Elle comporte un nombre important de membres déclinant une gamme d'appréciations mutuelles très variable : les rivalités et les défis y sont réguliers. Ce mode de fonctionnement provoque des conflits entre le groupe et l'out-group. Nixon, un adolescent de la Corpo raconte: "J'étais au quartier et je suis tombé en vélo devant la Corpo au complet. Après les collègues ils se foutaient de moi et y'avait deux types sur la route qui se marraient aussi. Gangsta et Mouloud, ils viennent me dire "Quoi tu te laisses vanner par des bouffons? ». Alors, je suis parti voir les deux gars et j'ai tapé un coup de pression. L'autre je l'ai déchiré, après on les a dépouillés ».

Ce qui ressemble de l'extérieur à un vol avec violence froidement prémédité s'avère un concours de circonstances où la honte, provoquée par le contrôle social de la bande, conduit au défi et à des violences physiques puis à un vol. Un autre élément explique l'engrenage décrit par Nixon : il fréquente la bande la plus délinquante de sa classe d'âge et pour être à la hauteur, il doit se bagarrer plus fréquemment que s'il traînait avec la bande du haut ou la DB. Sur Paris nord, un voisin, Manu, est entré en conflit avec un jeune de la rue, Steven. Son chien était l'objet du litige : il courait en liberté sans muselière et a bondi sur Steven qui a eu très peur. Manu n'a pas véritablement formulé d'excuses, suspectant Steven d'avoir adopté un comportement provocateur. Trois jours plus tard, quatre jeunes l'ont agressé et l'ont envoyé à l'hôpital. Steven a bénéficié de l'aide de David, de Malek et de Houcine. L'action n'était pas préparée: c'était l'opportunité en attente de Steven. David, Malek et Houcine étaient simplement présents au bon endroit et au bon moment. Ils n'avaient jamais eu de contact avec Manu, ce qui ne les a pas empêchés de le blesser. Houcine se justifie : "On était en train de galérer et on a vu Steven tout speed qu'est venu nous brancher. On peut pas dire non et puis ce cave le méritait bien. C'était une histoire à cause de son chien ».

Lorsque Houcine dit "on était en train de galérer », il explique par-là que cette bagarre a eu l'avantage de briser l'ennui. Lorsqu'il déclare « on a vu Steven tout speed qu'est venu nous brancher. On peut pas dire non », il nous laisse deviner le devoir de protection mutuelle qui le lie à Steven, ainsi que, peut-être, la peur que celui-ci lui inspire. Dans tous les cas, la logique d'appartenance à la bande suppose ce genre d'engagement. Enfin, lorsqu'il dit « et puis ce

25 Voir par exemple : Bruno Aubusson de Cavarlay, «La place des mineurs dans la délinquance enregistrée », 
cave le méritait bien. C'était une histoire à cause de son chien », il termine sa justification par un principe moral largement partagé, donc compréhensible par le plus grand nombre : le droit de se défendre en cas d'agression. Par ce biais, il rend compte de son adhésion à des principes moraux qui transcendent l'adhésion au groupe de pairs : il ne ferait pas n'importe quoi par solidarité avec les amis de la rue.

Une dimension ethnique peut jouer un rôle dans la formation des bandes, sans constituer un facteur décisif. Ainsi, la majorité des $\mathrm{CB}$ se réfère à la culture maghrébine. Expressions, coutumes, normes et valeurs (notamment sur la question du porc) favorisent l'intégration des jeunes de même origine. Gérard Mauger 26 constate : «le recrutement des bandes cooptées dans le cadre d'une même unité spatiale sur la base d'affinités de dispositions et de conditions peut passer pour une cooptation «ethnique », du fait de la visibilité des marqueurs corporels qui les désignent comme immigrés ou fils d'immigrés. Mais il est vrai qu'une «ethnicité » vague qui associe à telles propriétés corporelles (la couleur de la peau, les cheveux frisés, les yeux bridés, etc.) une culture ad hoc faite de bric et de broc, d'éléments hétéroclites et décontextualisés ( « renois »/《r rebeux », « céfrans »/《rebeux», «toubabs »/《renois », etc.) peut servir de ressource identitaire aux agents les plus démunis de ressources économiques et culturelles et s'intégrer dans la logique guerrière du monde des bandes, logique du territoire, du patronyme, des couleurs, de l'opposition «nationaux/étrangers », etc. ».

Une configuration relative à la sociologie de l'exclusion de Norbert Elias et John L. Scotson (1997) s'observe également. En fonction du turn-over résidentiel, elle distingue les natifs des lieux des derniers arrivés, comme c'est le cas entre la Bédo Connexion et la bande à Embre.

À l'adolescence, les «équipes » se connectent ou s'articulent davantage, les bandes s'élargissent, des petits nouveaux apparaissent, des jeunes qui, jadis, ne fréquentaient pas l'espace public, comme les lycéens de la bande du haut. Des copains en intègrent d'autres qui eux-mêmes en présentent de nouveaux. Cette dynamique s'apparente à un effet «boule de neige », la qualité du leadership de chaque bande en détermine la force car les bandes nécessitent des représentants charismatiques, et surtout une «figure de proue » derrière laquelle s'abriter. La rivalité ou l'association entre les principaux leaders d'une même classe d'âge produit «l'offre de bandes » propre à cette classe d'âge. Le fait que les rivalités au sommet de la structure hiérarchique expliquent «l'offre de bandes » correspond à un schéma

Les cahiers de la sécurité intérieure, ${ }^{\circ} 29,1997$.

26 Gérard Mauger, «Disqualification sociale, chômage, précarité et montée des illégalismes », Regards Sociologiques, $\mathrm{n}^{\circ} 21,2001$. 
qui s'appliquait déjà dans le vieux Boston italo-américain de William Foote Whyte ${ }^{27}$ : «Quand le chef change, ce n'est pas l'effet d'une révolte de la base mais d'une modification des relations au sommet de la structure. Lorsqu'un groupe se scinde en deux bandes, c'est qu'un conflit a opposé le chef à l'un de ses anciens lieutenants ». Cependant, le reste du groupe ne reste pas passif, une partie soutient tel ou tel leader au détriment de tel autre, de manière explicite ou implicite, sur le plan physique, symbolique et économique.

Exemple :

Sur Paris sud, un ancien KBA réapparaît après plusieurs années d'absence : Carlos mesure presque deux mètres et ne pèse pas moins de cent vingt kilos, il travaille pour son père, un petit entrepreneur. Costaud, bagarreur, expérimenté, malin, tchatcheur et généreux, il s'impose «naturellement», avec sa Mercedes, comme un meneur parmi les KBA, ce qui énerve le leader historique de cette bande, Farid. Ce dernier, face à la stature du personnage, reste silencieux, observe et attend. Peu à peu Carlos devient moins sympathique, il n'offre plus de tournées générales et se montre autoritaire. Farid y voit l'opportunité de regagner sa place. Il mobilise discrètement son réseau d'alliances : d'abord Mourad, un jeune craint et respecté, puis Faouzy. Un soir, Hicham, giflé par Carlos pour une simple faute de bon sens dans une partie de tarot, vient silencieusement grossir les rangs de la coalition. Lors d'un barbecue dans un petit parc à côté de la cité, Carlos, chargé de ramener la viande, arrive avec deux heures de retard. Farid saisit cette occasion. Mourad, Hicham et Faouzy le suivent. Le ton monte, comme d'autres gars de la bande, je prends mes distances : Carlos, blessé, regagne sa voiture en titubant et disparaît définitivement.

Dans le cas présent, la théorie de William Foote Whyte (1995) selon laquelle les modifications majeures de la structure hiérarchique d'une bande renvoient forcément à un conflit au sommet, n'analyse que la partie immergée de la réalité sociale, car si l'opposition Farid/Carlos confirme cette théorie, le reste de la bande joue néanmoins un rôle décisif : sans ses alliés, Farid n'aurait jamais pu chasser Carlos.

La force du nombre induit un léger caractère démocratique, elle oblige à prendre en compte l'opinion d'autrui dès lors que celui-ci se trouve en capacité de participer aux combats futurs ou d'influencer leurs résultats d'une manière ou d'une autre.

Toutes les bandes rencontrées sont hiérarchisées, mais cette hiérarchisation n'est pas forcément très précise, ne s'encombre pas de formalisme et peut être remise en cause par des concurrents soutenus ou non par la base. La hiérarchisation n'est pas toujours explicitée car

27 William Foote Whyte, Street Corner Society, la structure sociale d'un quartier italo-américain, Paris, La 
elle ne doit pas contredire abusivement le caractère libertaire et amical, imprégné d'égalitarisme, des réseaux de pairs en question. Son explicitation trop formelle blesserait l'orgueil viril des rivaux et celui des jeunes dominés qui préfèrent taire les humiliations de leur position. L'utilisation de la violence symbolique et physique doit être mesurée et doit conserver une part minimale de prévisibilité. Bien que cruelle et cynique, la vie de rue doit préserver un minimum de solidarité, de respect et de compréhension mutuelle pour ne pas devenir un univers complètement anomique, aucun des jeunes ne le souhaite. En conséquence, les garçons qui incarnent le leadership s'imposent par la ruse et la force mais refusent le titre de chef tout en jouissant de tous ses avantages.

Les classes d'âge : les « grands », les « jeunes » et les « petits»

Sur Paris Nord, l'unique bande de jeunes adultes se prénomme du nom de la cité et ajoute «Clica Una ». Clica est un terme espagnol qui peut signifier «bande ». Mohamed et Samir, leaders charismatiques, forment son pôle d'attraction. Ils se rassemblent dans un hall ou une cave aménagée à côté des commerces du quartier. Cette bande s'engage dans des pratiques diversifiées : trafics, consommation de drogues, activités sportives et artistiques. La vingtaine de jeunes adultes qui «traînent »s'y rassemble. Du fait de la petite taille de la cité, la moindre tentative d'évitement se repère facilement et déclenche la curiosité du groupe principal, incarné par la Clica Una.

Il s'agit d'une caractéristique de Paris nord : bande et classe d'âge ne forment qu'un seul et unique espace de socialisation, que cela soit chez les jeunes adultes, les adolescents ou les enfants.

La «bande-classe d'âge » adolescente s'appelle « Nicky Crew ». Crew, un terme anglais, peut signifier «bande ». « Nicky » renvoie à Nike, la marque de leurs vêtements. Une vingtaine de garçons se retrouve dans un hall situé à l'abri du regard des grands frères, jugés autoritaires. Leur groupe tend à se spécialiser dans la consommation de cannabis. Le lien social créé par cette consommation a réuni deux réseaux relationnels qui, à l'origine, étaient clairement distincts. On observe également une forme de déviance générationnelle: Abdelkader et Alexandre, âgés respectivement de douze et onze ans, préfèrent fréquenter des enfants. 
La «bande-classe d'âge » des enfants s'appelle justement « la bande d'Abdelkader». Son point de rencontre se situe à l'écart des plus âgés, ou sur le terrain de sport, au centre de la cité. La peur qu'elle inspire aux autres enfants les éloignent de la rue.

La répartition spatiale des différentes classes d'âge apparaît ici flagrante : la cité tend à se découper en trois zones, chaque zone correspondant au territoire de référence d'une classe d'âge, chaque zone se soustrayant au contrôle visuel exercé par ceux qui occupent les deux autres. Chacun se débarrasse ainsi de ses petits frères, qui sinon «mouchardent », ou de ses grands frères, qui sinon donnent des ordres. Le terrain de sport, situé au centre, symbolise le point de ralliement.

Sur Paris nord, les «bandes-classes d'âge » rassemblent en permanence les garçons de la même tranche d'âge. Sur Paris sud et Marseille, les classes d'âge jouent également ce rôle mais le font de manière irrégulière, et constituent des espaces relationnels où la confiance devient plus aléatoire en comparaison de celle qui unit les membres d'une bande.

La classe d'âge correspond à une «génération » ou plus exactement à une demi-génération avec au maximum dix ans d'écart. Sur Paris sud et Marseille, elle se nourrit des activités qui nécessitent le maximum de personnes et qui impliquent en conséquence une certaine démesure par rapport au quotidien : jeux géants de «cache-cache » pour les enfants, grands tournois de football pour les adolescents, ambiances festives ou guerrières au jeune âge adulte. Sur Paris sud, pour combattre les bandes des cités rivales, l'union s'impose. Ces alliances temporaires réalisées dans le but d'attaquer un ennemi commun s'observaient dans les années 1960 à plus grande échelle. Jean Monod ${ }^{28}$ constatait que les bandes de loubards du nord de Paris se regroupaient pour affronter les coalitions banlieusardes issues de La Courneuve et de Pantin.

Le business participe également à l'ouverture sur l'ensemble des pairs, à l'instar de la lassitude relationnelle qui s'installe dans les bandes. Certains jeunes prennent de véritables «bouffées d'oxygène » en modifiant temporairement leurs habitudes relationnelles. Le plus souvent ils se dirigent vers un groupe de la même classe d'âge, mais peuvent aussi côtoyer un groupe plus jeune ou plus vieux, ce qui représente alors une forme de promotion sociale. L'intégration à une bande plus âgée peut correspondre à un choix « carriériste », en particulier lorsqu'il s'agit de carrière délinquante, mais le lecteur ne doit pas s'imaginer une organisation sophistiquée ou rigide, avec des réseaux clairement délimités, avec des épreuves et des stades précis qui marquent le passage dans la catégorie supérieure. Je décris des tendances

\footnotetext{
28 Jean Monod, Les Barjots. Essai d'ethnologie des bandes de jeunes, Paris, Julliard, 1968.
} 
organisationnelles et non une division radicale de l'espace social. Il existe par exemple des jeunes sans équipe qui fréquentent l'ensemble des bandes de leur classe d'âge, comme des «électrons libres». Certains ne donnent pas leur confiance, côtoient tout le monde et personne en particulier, sont solitaires bien que constamment entourés.

Contrairement à la thèse défendue par David Lepoutre dans le remarquable «Cœur de banlieue ${ }^{29}$, le « Nous » précaire et temporaire (car exclusivement «juvénile ») ici étudié ne se réduit pas à une dimension adolescente, mais repose de plus en plus sur des liens « intergénérationnels » qui courent de l'enfance jusqu'à 30 voire 35 ans, jusqu'à la fin d'une jeunesse interminable. Les loubards, absorbés rapidement par le monde de l'usine, sortaient beaucoup plus tôt de la vie juvénile de rue, du «monde des bandes », en passant plus rapidement de la famille parentale à la famille conjugale. Aujourd'hui, du fait des difficultés de l'accession au logement, elles-mêmes liées aux impasses de l'insertion professionnelle, ce passage est retardé et la vie juvénile de rue intègre de plus en plus l'expérience et les initiatives du jeune âge adulte, en particulier en ce qui concerne la problématique de la délinquance organisée.

L'adolescence symbolise néanmoins incontestablement le temps fort de ce «Nous ». Les adolescents découvrent la vie juvénile de rue dans sa globalité et sa complexité, ils ne s'en lassent pas, ils y croient, contrairement aux jeunes adultes qui cherchent sans succès une « porte de sortie ».

Les différences d'âge correspondent à des repères importants de la structure hiérarchique. La remise en cause de ce principe de hiérarchisation ne passe donc pas inaperçue. Les plus vieux se plaignent du caractère entreprenant des plus jeunes, et les plus jeunes du caractère autoritaire des plus vieux. Il existe une lutte entre les «entrants» et les «sortants ». Une partie des relations intergénérationnelles se résume ainsi au maintien d'une ségrégation territoriale. La situation se dégrade lorsque la différenciation «jeunes/vieux » se superpose à la distinction «natifs des lieux/étrangers » produite par le turn-over résidentiel, comme c'est le cas sur Paris sud entre la bande à Embre et les CB.

\section{4) Conclusion}

Malgré le fait qu'elle ne représente que $10 \%$ de la population masculine âgée de moins de trente ans, la vie juvénile de rue et de cité détient une place prédominante dans la vie sociale des cités étudiées et semble parfois, dans certains endroits et à certaines heures, propriétaire

\footnotetext{
29 David Lepoutre, Cour de banlieue, codes, rites et langages, Paris, Odile Jacob, 1997.
} 
de l'espace public. Elle se compose de différents réseaux relationnels dont l'importance quantitative varie considérablement, passant de deux ou trois individus en ce qui concerne «l'équipe » à plusieurs dizaines en ce qui concerne « la bande » ou la « classe d'âge ». Sur le plan qualitatif, nous passons facilement de relations entre pairs à des rapports intergénérationnels. Cet ensemble de liens de concurrence et de solidarité explique la force de la vie juvénile de rue lorsqu'elle se rassemble et s'unit contre un ennemi commun. Le quartier, comme cela a déjà été constaté à l'époque des apaches et des loubards, constitue un espace de référence qui permet d'articuler ces réseaux jusqu'à ce qu'ils forment, à des moments spécifiques (sportifs, festifs ou guerriers par exemple), un seul et unique groupe.

Ces réseaux de pairs se spécialisent à partir de l'adolescence (délinquance d'expression ou d'acquisition, activités sportives, voire religieuses ou artistiques, consommation de drogues dures ou douces). Cette spécialisation crée des échanges de savoir-faire mais impose également des «droits d'entrée » spécifiques : il s'agit de montrer son adéquation avec la spécialisation du groupe, c'est-à-dire avec son activité centrale et le mode de vie qui en découle.

L'intégration de nouveaux membres s'effectue parfois sur la base d'une différenciation «natifs des lieux/étrangers » conditionnée par le turn-over résidentiel. Le critère «ethnique » apparaît contingent.

Ces réseaux ne sont pas systématiquement délimités de façon claire malgré les droits d'entrée spécifiques qu'ils tendent à imposer, et ne fonctionnent pas en permanence. Ils peuvent se confondre en partie et céder la place à des modes aléatoires de rencontre. Ils se décomposent et se recomposent mais bénéficient d'une stabilité et d'une lisibilité suffisantes pour organiser sur le long terme les pratiques et les représentations propres à la vie juvénile de rue et de cité. Certains s'avèrent purement utilitaristes, d'autres lourdement chargés sur le plan affectif.

Si la loi du plus fort hiérarchise ces acteurs et groupes d'acteurs, nous observons néanmoins un léger caractère démocratique grâce à l'influence déterminante de la force du nombre : celle-ci induit le développement de dispositions tactiques et politiques. 